\title{
Dípteros minadores e seus parasitóides em plantas de crescimento espontâneo em pomar orgânico de citros em Montenegro, RS, Brasil
}

\author{
Janaína Pereira dos Santos ${ }^{1}$, Luiza Rodrigues Redaelli² \& Fábio Kessler Dal Soglio ${ }^{2}$
}

1. Estação Experimental de Caçador, Laboratório de Entomologia, EPAGRI, Caixa Postal 591, 89500-000 Caçador, SC, Brasil. (janapereira@epagri.rct-sc.br)

2. Departamento de Fitossanidade, UFRGS, Av. Bento Gonçalves, 7712, 91540-000 Porto Alegre, RS, Brasil. (luredael@ufrgs.br; fabiods@ufrgs.br)

\begin{abstract}
Diptera leafminers and their parasitoids in spontaneous vegetation in organic citrus orchard in Montenegro, RS, Brazil. Leafminers and their parasitoids communities analysis is necessary to supply information about the biotic regulation and to maintenance of the biodiversity in the agroecosystem. This study aimed to register Diptera leafminers and their parasitoids, present in the vegetation spontaneously growing at the citrus orchard from May 2003 to May 2004. The work was conducted in Montenegro, RS, in an organic orchard of the hybrid 'Murcott'. Samplings were taken fortnightly, collecting in each occasion all the plants with mines found in an area delimited by a $0.28 \mathrm{~m}^{2}$ arc thrown in the lines and between lines of 30 randomly chosen trees. In the lab, the number of larvae and pupae per leaf of Diptera leafminers were recorded. Throughout the study, it was found 15 species of Diptera leafminers, 15 species of leafminer host plants (distributed in six families) and 15 species of micro hymenopterans parasitoids (distributed in three families). It was verified that the Diptera leafminers had presented great specificity to their host plants; therefore, the appropriate management of the spontaneous vegetation of the orchards may favor the establishment and multiplication of natural enemies of these leafminers insects.
\end{abstract}

KEYWORDS. Diptera, Agromyzidae, micro hymenopterans, host records.

RESUMO. A análise de comunidades minadores e seus parasitóides é importante para a compreensão da regulação biótica e para a manutenção da biodiversidade em agroecossistemas. Este trabalho teve como objetivos registrar os dípteros minadores e seus parasitóides na vegetação de crescimento espontâneo de um pomar orgânico de citros, de maio de 2003 a maio de 2004. O trabalho foi conduzido no município de Montenegro, RS, em um pomar do híbrido tangor 'Murcott'. Realizaram-se amostragens quinzenais, coletando-se em cada ocasião todas as folhas contendo minas presentes na área delimitada por um aro de $0,28 \mathrm{~m}^{2}$, que era jogado nas linhas e nas entrelinhas de 30 árvores sorteadas. No laboratório registrou-se o número de larvas e pupas de dípteros minadores por folha. Foram registradas 15 espécies de dípteros minadores, 15 espécies de plantas hospedeiras (distribuídas em seis famílias) e 15 espécies de microimenópteros parasitóides (distribuídas em três famílias). Os dípteros minadores apresentaram grande especificidade às suas plantas hospedeiras. Portanto, o manejo adequado desta vegetação pode favorecer o estabelecimento e a multiplicação de inimigos naturais destes insetos minadores.

PALAVRAS-CHAVE. Diptera, Agromyzidae, microimenópteros, registro de hospedeiros.

Dentre os grupos que incluem insetos com hábito minador, destaca-se Diptera. Nesta ordem, a principal família com este hábito é Agromyzidae, com representantes principalmente nos gêneros Liriomyza e Agromyza (HespenheIDE, 1991; B Yers, 2002; CARLETTI, 2004). Segundo Gallo et al. (2002), várias espécies de agromizídeos estão associadas a plantas cultivadas, entretanto, Byers (2002) relata que muitos podem ser pragas secundárias em outras culturas e atacar plantas espontâneas.

Diversos parasitóides são associados às moscas minadoras. No Brasil, Pereira et al. (2002) mencionam Opius sp. (Hymenoptera: Braconidae) parasitando Liriomyza huidobrensis (Blanchard, 1926). Na Argentina, VALLADARES \& SALVo (2001) registraram 29 espécies de moscas minadoras pertencentes a Agromyzidae e 46 espécies de microimenópteros parasitóides associados. Na Colômbia, CuRE \& CANTOR (2003) observaram o braconídeo, Diglyphus begini (Ashmead, 1904) associado à mosca minadora L. huidobrensis. Em Cuba, León et al. (2000) registraram Diglyphus sp. sobre Liriomyza sp. Nos Estados Unidos da América, SCHUSTER et al. (1991) encontraram Opius dissitus Muesebeck, 1963; O. bruneipes Gahan, 1913 e O. mandibulares Gahan, 1945 parasitando Liriomyza spp. e PATEL et al. (2003) relataram D. intermedius (Girault, 1917) sobre L. trifolli (Burgess, 1880). No Canadá, VenetTe et al. (2003) relatam Diglyphus spp. associado a várias espécies de Liriomyza.

Sabe-se que os organismos expostos a conjuntos diversos de condições e recursos respondem de forma diferente, evidenciando um tamanho populacional e uma dinâmica variável, tornando-se, portanto, importante o estudo destes, nos vários locais em que ocorrem. A descrição da variação temporal da abundância é aspecto importante para a identificação e o entendimento dos fatores que influenciam a flutuação de uma população (Begon \& Mortimer, 1986). O conhecimento desses processos permite a realização de previsões a respeito das flutuações populacionais, o que pode ser utilizado no manejo de populações, com o objetivo de conservação ou para o controle de populações eruptivas (BEGON \& MORTIMER, 1986; Begon et al., 1990). O presente trabalho teve como objetivo registrar a distribuição dos dípteros minadores e dos parasitóides associados à vegetação de 
crescimento espontâneo, em um pomar orgânico de citros, contribuindo desta maneira, para a compreensão de aspectos da regulação biótica e da manutenção da biodiversidade em agroecossistemas.

\section{MATERIAL E MÉTODOS}

O trabalho foi desenvolvido no município de Montenegro (2968'S e 51 $46^{\prime} \mathrm{W}$ ), localizado no vale do rio Caí, RS. A topografia da região é levemente ondulada, com menos de $100 \mathrm{~m}$ de altitude, e pertence à região da Depressão Central. Os solos são profundos e de textura argilosa (Unidade Bom Retiro). A temperatura média anual é de $19,4^{\circ} \mathrm{C}$, apresentando chuvas abundantes $(1.537 \mathrm{~mm} /$ ano) e bem distribuídas (Rodriguez et al., 1991).

Os registros diários dos dados meteorológicos referentes à temperatura máxima, mínima e média, precipitação e umidade relativa do ar foram fornecidos pela Estação Experimental da FEPAGRO (Fundação Estadual de Pesquisa Agropecuária), situada no município de Taquari, RS (distante cerca de $30 \mathrm{~km}$ do local de estudo). Com base nestes registros, calcularam os valores médios mínimos e máximos destes fatores para cada estação (Tab. I).

As coletas foram realizadas em pomar de tangoreiro Murcott (Citrus sinensis x Citrus reticulata), enxertado sobre Poncirus trifoliata, com área de 0,6 ha e cerca de 370 plantas com 12 anos de idade. $\mathrm{O}$ espaçamento entre plantas é de 3,5 m e nas entrelinhas é de $5 \mathrm{~m}$.

Quinzenalmente, de maio de 2003 a maio de 2004, foram realizadas amostragens na vegetação que cresce espontaneamente entre as plantas de citros e nas entrelinhas. Para o sorteio dos pontos, as plantas de citros foram numeradas e, através do programa de números aleatórios BioEstat (AYres et al., 2000), em cada ocasião sortearam-se números que corresponderiam aos pontos amostrais. Em cada ponto sorteado recolheu-se uma unidade de amostra na linha e outra na entrelinha. Retirouse em cada ocasião 60 unidades de amostra, que consistiram de todas as folhas com minas presentes num círculo de $0,28 \mathrm{~m}^{2}$, delimitado por um aro de PVC com 60 cm de diâmetro, adaptação feita do método do quadrilátero proposto por SoutHwoOd (1978).

Para retirar a planta inteira utilizou-se uma pá de jardineiro, e quando isto não era possível retiraram-se apenas os ramos com o auxílio de uma tesoura de poda. As plantas e/ou ramos recolhidos foram colocados, individualmente, em sacos plásticos etiquetados, com data de amostragem, número da planta de citros e identificação da posição (entre plantas ou entrelinha). Os sacos plásticos foram acondicionados em caixa de isopor contendo termogel para o transporte até o laboratório.

No laboratório, as folhas foram examinadas com o auxílio de microscópio estereoscópio, registrando-se o número de larvas e/ou pupas de dípteros minadores. Posteriormente, as folhas de cada espécie vegetal foram acondicionadas, individualmente, em placas de Petri de 9 cm de diâmetro e 1,5 cm de altura ou em caixas "gerbox" de 11,2 cm de diâmetro e 3,4 cm de altura, as quais foram mantidas em câmara climatizada (fotofase de 12 horas, $25^{\circ} \mathrm{C} \pm 1^{\circ} \mathrm{C}$ ) até a emergência dos adultos de minadores e/ou de parasitóides. Para que as folhas permanecessem túrgidas por um período maior, colocou-se no pecíolo das mesmas, um chumaço de algodão umedecido, molhado diariamente para favorecer o desenvolvimento completo dos insetos. Com base nos adultos emergidos foi possível associar as espécies com os imaturos coletados.

Os parasitóides e os dípteros emergidos foram conservados individualmente em recipientes tipo "ependorff" contendo álcool 70\%, e enviados a especialistas para identificação.

A identificação em nível de família dos parasitóides foi efetuada com o auxílio da chave dicotômica de GAULD \& Hanson (1995) e em nível genérico e/ou específico por especialistas.

Fez-se uma coleção-referência dos insetos coletados (dípteros minadores e seus parasitóides), a qual se encontra no Departamento de Fitossanidade, Faculdade de Agronomia da Universidade Federal do Rio Grande do Sul (UFRGS). Os exemplares que foram enviados para os especialistas encontram-se nas coleções-referência dos mesmos.

Foram feitas exsicatas das plantas nas quais foram encontrados os dípteros minadores, estas foram identificadas com o auxílio da bibliografia (SMITH \& Downs, 1972; Cabrera \& Klein, 1989; Kissmann \& Groth, 2000a,b,c; W3Tropicos, 2004) e por comparação com o acervo de plantas do herbário do Departamento de Botânica da UFRGS. Essas exsicatas estão depositadas no Departamento de Fitossanidade da UFRGS.

\section{RESULTADOS E DISCUSSÃO}

Durante as 27 ocasiões de amostragem, coletaramse 521 larvas e 107 pupas de dípteros minadores e registrou-se a emergência de 140 indivíduos, todos pertencentes a Agromyzidae (incluídos em 15 espécies e distribuídos nos gêneros Calycomyza, Liriomyza e Agromyza). Foram obtidos 134 indivíduos parasitóides, todos pertencentes a Hymenoptera, distribuídos em 15 espécies de três famílias: Eulophidae, Braconidae e Figitidae (Tab. II).

No presente estudo verificou-se que a maioria das espécies de minadores foi registrada em apenas uma espécie de planta hospedeira sugerindo uma alta especificidade minador/planta (Tab.II). Já em relação aos parasitóides, não houve alta especificidade com o hospedeiro (Tab. II).

Tabela I. Valores médios de temperatura mínima, máxima, precipitação e umidade relativa do ar registrados nas estações do ano de maio de 2003 a maio de 2004 em Taquari, RS.

\begin{tabular}{lcccc}
\hline Estação & $\begin{array}{c}\text { Temperatura } \\
\text { mínima } \\
\left({ }^{\circ} \mathrm{C}\right)\end{array}$ & $\begin{array}{c}\text { Temperatura } \\
\text { máxima } \\
\left({ }^{\circ} \mathrm{C}\right)\end{array}$ & $\begin{array}{c}\text { Precipitação } \\
\text { pluviométrica } \\
(\mathrm{mm} / \text { dia })\end{array}$ & $\begin{array}{c}\text { Umidade } \\
\text { relativa } \\
\text { do ar }(\%)\end{array}$ \\
\hline Outono (2003) & 8,9 & 20,3 & 5,6 & 84,8 \\
Inverno (2003) & 8,8 & 20,5 & 2,7 & 71,0 \\
Primavera (2003) & 15,3 & 25,9 & 7,6 & 76,0 \\
Verão (2003 para 2004) & 18,2 & 29,0 & 0,8 & 79,3 \\
Outono (2004) & 15,5 & 26,9 & 3,0 & 81,5 \\
\hline
\end{tabular}


Tabela II. Dípteros minadores e seus respectivos parasitóides presentes em plantas de crescimento espontâneo em um pomar de 'Murcott', Montenegro, RS entre maio de 2003 e maio de 2004. "Não emergiram adultos; *Provavelmente trata-se de Liriomyza baccharidis Spencer, 1963; **Provavelmente trata-se de Calycomyza eupatorivora Spencer, 1963; ***Provavelmente trata-se de Calycomyza hyptidis Spencer, 1963.

\begin{tabular}{|c|c|c|}
\hline Dípteros minadores & Parasitóides & Plantas hospedeiras \\
\hline Família/Espécie & Família/Espécie/Número de emergidos & Família/Espécie/Nome comum \\
\hline AGROMYZIDAE & EULOPHIDAE & POACEAE \\
\hline Agromyza sp. & Chrysocharis sp. 1 (2) & Brachiaria decumbens (capim braquiária) \\
\hline \multirow[t]{8}{*}{ Calycomyza sp. 1} & BRACONIDAE- Centistidea sp. 1 (2); Opius & ASTERACEAE \\
\hline & sp. $1(2) ;$ Opius sp. 2 (6); Opius sp. 4 (1) & Baccharis anomala (cambará-de-cipó) \\
\hline & EULOPHIDAE- Chrysocharis sp. 1 (4); & Baccharis anomala \\
\hline & Chrysocharis sp. 2 (1); Chrysocharis tristis & \\
\hline & Hansson, 1987 (1); Chrysocharis vonones & \\
\hline & Walker, 1839 (1); Closterocerus coffeellae & \\
\hline & Lhering, 1914 (4) & \\
\hline & $\begin{array}{l}\text { FIGITIDAE- Agrostocynips clavatus Diaz, } \\
1976 \text { (3) }\end{array}$ & Baccharis anomala \\
\hline Calycomyza sp. 2 & Sem registro de parasitóides & Bidens pilosa (picão-preto, picão-campo) \\
\hline Calycomyza sp. $3^{\bullet}$ & BRACONIDAE- Opius sp. 1 (4) & Erechtites valerianifolia (capiçoba) \\
\hline Calycomyza sp. $4^{* *}$ & Sem registro de parasitóides & Eupatorium inulifolium (eupatório, cambará) \\
\hline \multirow[t]{2}{*}{ Calycomyza sp. $5 * * *$} & EULOPHIDAE- Chrysocharis sp. 1 (1); & LAMIACEAE \\
\hline & Chrysocharis sp. 2 (1) & Hyptis mutabilis (sambacaitá, bamburral) \\
\hline \multirow{4}{*}{$\begin{array}{l}\text { Calycomyza ipomoea } \\
\text { (Frost, 1931) }\end{array}$} & BRACONIDAE & CONVOLVULACEAE \\
\hline & Opius sp. 2 (6); Opius sp. 1 (1) & Ipomoea cairica \\
\hline & $\begin{array}{l}\text { EULOPHIDAE- C. vonones (3); C. coffeellae (2); } \\
\text { Closterocerus sp.(2); Chrysocharis sp. } 1 \text { (2); } \\
\text { Neochrysocharis } \mathrm{sp} .(1)\end{array}$ & Ipomoea cairica (corriola, corda-de-viola) \\
\hline & FIGITIDAE- A. clavatus (13) & Ipomoea cairica \\
\hline Calycomyza ipomoea & Sem registro de parasitóides & Ipomoea purpurea (campainha, jetirana) \\
\hline \multirow{4}{*}{$\begin{array}{l}\text { Calycomyza malvae } \\
\text { (Burgess, 1880) }\end{array}$} & & MALVACEAE \\
\hline & BRACONIDAE- Opius sp. 2 (1) & Sida urens (guanxuma, vassourinha) \\
\hline & $\begin{array}{l}\text { EULOPHIDAE- Chrysocharis sp. } 2 \text { (5); } \\
\text { Chrysocharis sp. } 1 \text { (4); C. tristis (1) }\end{array}$ & Sida urens \\
\hline & FIGITIDAE- A. clavatus (14) & Sida urens \\
\hline Calycomyza mikaniae & FIGITIDAE- A. clavatus (2) & ASTERACEAE \\
\hline Spencer, 1973 & & Mikania micrantha \\
\hline \multirow{3}{*}{$\begin{array}{l}\text { Calycomyza sidae } \\
\text { Spencer, } 1973\end{array}$} & Sem registro de parasitóides & MALVACEAE \\
\hline & & Sida rhombifolia (guanxuma, guanxuma-preta) \\
\hline & & ASTERACEAE \\
\hline \multirow[t]{2}{*}{ Liriomyza sp. $1^{*}$} & BRACONIDAE- Centistidea sp. 2 (1) & Baccharis anomala \\
\hline & EULOPHIDAE- C. coffeellae (3) & Baccharis punctulata (cambará-cheiroso) \\
\hline \multirow[t]{2}{*}{ Liriomyza sp. 2} & BRACONIDAE- Opius sp. 3 (1) & Conyza bonariensis (buva) \\
\hline & EULOPHIDAE- C. coffeellae (2) & Conyza bonariensis \\
\hline \multirow[t]{2}{*}{ Liriomyza sp. 3} & Sem registro de parasitóides & AMARANTHACEAE \\
\hline & & Iresine diffusa (neve-da-montanha, paina) \\
\hline \multirow{5}{*}{$\begin{array}{l}\text { Liriomyza commelinae } \\
\text { Frost, } 1931\end{array}$} & BRACONIDAE- Opius sp. 1 (16); Centistidea & COMMELINACEAE \\
\hline & sp. 1 (2); Opius sp. 5 (1) & Commelina diffusa (trapoeraba, andaca) \\
\hline & $\begin{array}{l}\text { EULOPHIDAE- C. vonones (3); Chrysocharis } \\
\text { sp. } 2 \text { (2); C. tristis (1) }\end{array}$ & Commelina diffusa \\
\hline & FIGITIDAE- A. clavatus (6) & Commelina diffusa \\
\hline & & ASTERACEAE \\
\hline Liriomyza mikaniae & BRACONIDAE- Opius sp. 2 (4) & Mikania micrantha (guaco, micania) \\
\hline Spencer, 1977 & FIGITIDAE- A. clavatus (2) & Mikania micrantha \\
\hline
\end{tabular}


Verificou-se que Calycomyza sp. 1 ocorreu o ano todo (Fig. 1) entretanto, o maior número de larvas e pupas foi observado no inverno e na primavera (períodos em que também foram registrados parasitóides emergidos deste minador). No verão e no outono poucas larvas e pupas deste minador foram amostradas e tampouco se verificou a emergência de parasitóides. Dez diferentes espécies de parasitóides emergiram de larvas e/ou pupas de Calycomyza sp. 1, sendo a maioria eulofídeos (Tab. II). Calycomyza sp. 1 foi encontrada apenas na asterácea Baccharis anomala que ocorre no sul do Brasil (BARROSO \& BuENo, 2002) durante o ano todo (o que explica, em parte, a coleta de larvas e pupas deste minador em todas as estações).

Calycomyza ipomoea (Frost, 1931) foi amostrada em praticamente todo o período, com exceção do inverno e início da primavera (Fig. 2). O mesmo foi observado em relação aos parasitóides associados com esta espécie. Oito espécies de parasitóides foram registradas associadas com este minador, a maioria delas de Eulophidae (Tab. II). Este minador esteve associado somente com as convolvuláceas Ipomoea cairica e $I$. purpurea, as quais ocorrem o ano inteiro (KISSMANN \& Groth, 2000a). Portanto, o fato de não terem sido coletados indivíduos no inverno pode estar relacionado à prevalência de condições climáticas não favoráveis para os indivíduos desta espécie neste período.

Calycomyza malvae (Burgess, 1880) ocorreu praticamente durante $\mathrm{o}$ ano todo, exceto em boa parte do inverno, bem como os seus parasitóides. No período em que o maior número de larvas e pupas do minador foi coletado (Fig. 3), obteve-se também o maior número de parasitóides, sendo estes distribuídos em cinco espécies (Tab. II). Este minador foi encontrado na malvácea Sida urens, espécie que ocorre o ano inteiro (Kissmann \& Groth, 2000b), o que explica a ocorrência de larvas e pupas em praticamente todo o período de estudo.

Verificou-se que Liriomyza commelinae Frost, 1931 foi encontrada em todas as estações, porém o número de indivíduos foi bastante variável entre as ocasiões (menor no inverno e no início do verão) (Fig. 4). As sete espécies de parasitóides associadas a esta última foram registradas nestas mesmas ocasiões (Fig. 4). A planta hospedeira deste minador foi uma comelinácea, Commelina diffusa, que ocorre o ano inteiro. Por conseguinte, a baixa freqüência deste minador em determinadas ocasiões pode estar relacionada com as condições climáticas. Esta espécie de mosca minadora foi citada por CHÁvez (2003) atacando as comelináceas $C$. diffusa, Commelina erecta e Commelina sp. na Guatemala.

Em relação a outras espécies de Liriomyza, verificou-se que Liriomyza sp. 1 foi amostrada em duas ocasiões no inverno (duas larvas), e em apenas uma ocasião na primavera (duas larvas), obtendo-se delas somente duas espécies de parasitóides (Tab. II). As plantas hospedeiras destes minadores foram as asteráceas $B$. anomala e $B$. punctulata, que podem ocorrer o ano inteiro, segundo BARRoso \& Bueno (2002). Desta maneira, acredita-se que as condições climáticas afetaram mais a ocorrência deste minador, do que a planta hospedeira.

Liriomyza sp. 2 foi encontrada sobre a asterácea Conyza bonariensis em apenas uma ocasião no inverno (18 larvas), duas na primavera (nove larvas) e uma no verão (duas larvas e uma pupa). Em Liriomyza sp. 2 também foram observadas apenas duas espécies de parasitóides (Tab. II). As coletas deste minador coincidiram com o período de ocorrência da planta, que germina com maior intensidade no final do outono e no inverno, terminando o ciclo na primavera ou no verão. Liriomyza sp. 3, que se encontrava sobre Iresine diffusa (Amaranthaceae) foi obtida em todas as estações (cinco larvas e duas pupas), porém, com baixa freqüência, apesar da planta hospedeira ocorrer o ano todo, de acordo com Sмiтh \& Downs (1972). Não foram registrados parasitóides nesse díptero.

Ao longo do período de estudo, adultos e larvas de Liriomyza mikaniae Spencer, 1977 foram registrados em apenas sete ocasiões e em pequena abundância, comparado às demais espécies de minadores. Em quatro

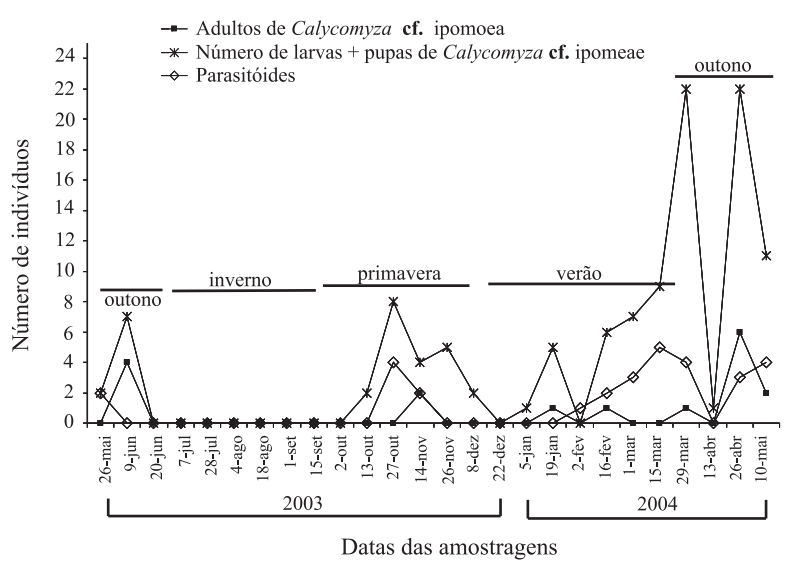

Fig. 2. Número de larvas + pupas de Calycomyza ipomoea amostradas em plantas de crescimento espontâneo, em pomar de tangor 'Murcott' e número de adultos de Calycomyza ipomoea e de parasitóides emergidos em laboratório, por ocasião de amostragem de maio de 2003 a maio de 2004, Montenegro, RS.
Fig. 1. Número de larvas + pupas de Calycomyza sp. 1 amostradas em plantas de crescimento espontâneo, em pomar de tangor 'Murcott' e número de adultos de Calycomyza sp. 1 e de parasitóides emergidos em laboratório, por ocasião de amostragem de maio de 2003 a maio de 2004, Montenegro, RS. 
destas ocasiões constatou-se também a presença de parasitóides (Fig. 5) de duas espécies (Tab. II). Observouse que a planta hospedeira deste minador, a asterácea Mikania micrantha foi pouco abundante no inverno e no outono, o que se deve à sua floração e frutificação esporádica durante o ano (CABRERA \& KLEIN, 1989). Assim, explica-se em parte o baixo número de indivíduos de $L$. mikaniae coletados nestas estações.

Em relação às demais espécies de Calycomyza, amostraram-se, no verão duas larvas de Calycomyza sp. 2 em Bidens pilosa (Asteraceae) (Tab. II), sendo que esta espécie vegetal ocorre durante todo o ano, porém, com maior intensidade no verão (KISSMANN \& GROTH, 2000a). Encontraram-se nove larvas de Calycomyza sp. 3 em Erechtites valerianifolia (Asteraceae) em apenas uma ocasião de amostragem (primavera) e apenas uma espécie de parasitóide associada (Tab. II). A baixa frequiência deste minador pode ser explicada pelo fato de que $E$. valerianifolia apresenta um período curto de

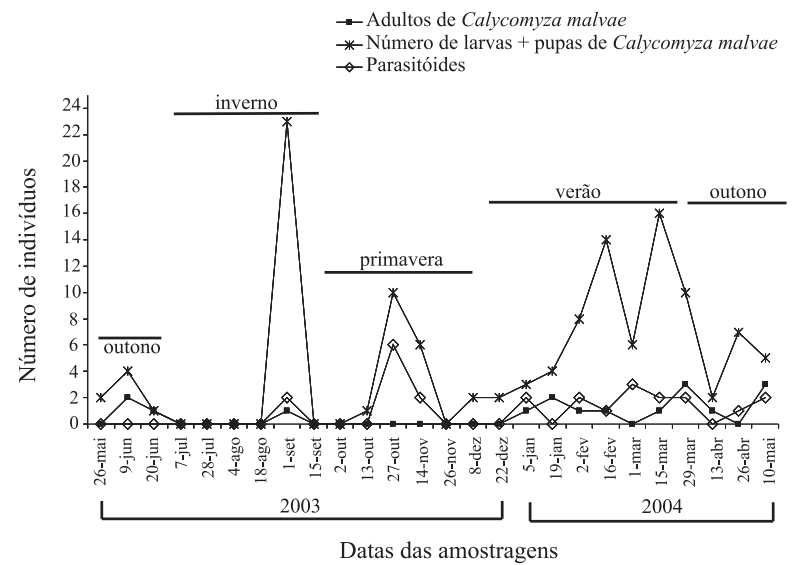

Fig. 3. Número de larvas + pupas de Calycomyza malvae amostradas em plantas de crescimento espontâneo, em pomar de tangor 'Murcott' e número de adultos de Calycomyza malvae e de parasitóides emergidos em laboratório, por ocasião de amostragem de maio de 2003 a maio de 2004, Montenegro, RS

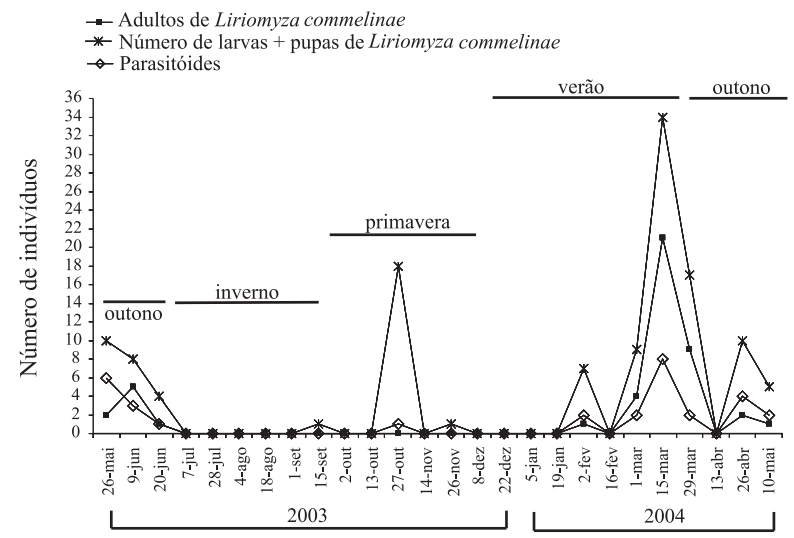

Datas das amostragens

Fig. 4. Número de larvas + pupas de Liriomyza commelinae amostradas em plantas de crescimento espontâneo, em pomar de tangor 'Murcott' e número de adultos de Liriomyza commelinae e de parasitóides emergidos em laboratório, por ocasião de amostragem de maio de 2003 a maio de 2004, Montenegro, RS. florescimento (aproximadamente 100 a 120 dias) (KISSMANN \& GROTH, 2000a).

Calycomyza sp. 4 foi encontrada em Eupatorium inulifolium (Asteraceae) esporadicamente em duas coletas no verão (sete larvas) e uma no outono (uma larva); ambos períodos coincidem com a ocorrência da planta hospedeira na região do estudo. Em Calycomyza sp. 4 não foram encontrados parasitóides. Já Calycomyza sp. 5 associada a Hyptis mutabilis (Lamiaceae) foi observada no verão (oito larvas e três pupas) e no outono (três larvas), das quais se obteve duas espécies de parasitóides.

Calycomyza mikaniae Spencer, 1973 ocorreu esporadicamente (um registro no final do outono, um no final do inverno e dois na primavera) e, da mesma maneira que para L. mikaniae, a planta hospedeira deste minador foi $M$. micrantha. Associado a $C$. mikaniae registrou-se uma espécie de parasitóide (Tab. II).

Foram obtidas apenas duas larvas de Calycomyza sidae Spencer, 1973 (uma no outono e outra no verão) e nenhum parasitóide foi registrado (Tab. II). A planta hospedeira deste minador foi a malvácea Sida rhombifolia, que tem ocorrência anual (KISSMANN \& GROTH, 2000b).

Agromyza sp. ocorreu em apenas duas ocasiões, uma no outono (duas larvas) e a outra no verão (uma larva) das quais foram obtidas duas espécies de parasitóides. Este minador foi coletado em Brachiaria decumbens (Poaceae), que segundo Kissmann \& Groth (2000c) é uma espécie perene.

Os resultados obtidos salientam a grande especificidade dos dípteros minadores com suas plantas hospedeiras corroborando o observado por SPENCER (1996) de que este grupo é, provavelmente, mais dependente da presença das plantas do que de condições climáticas.

Os dados aqui obtidos evidenciam a grande diversidade de espécies, tanto de dípteros minadores como de himenópteros parasitóides, muitas das quais se tratando, provavelmente, de novos registros e/ou de

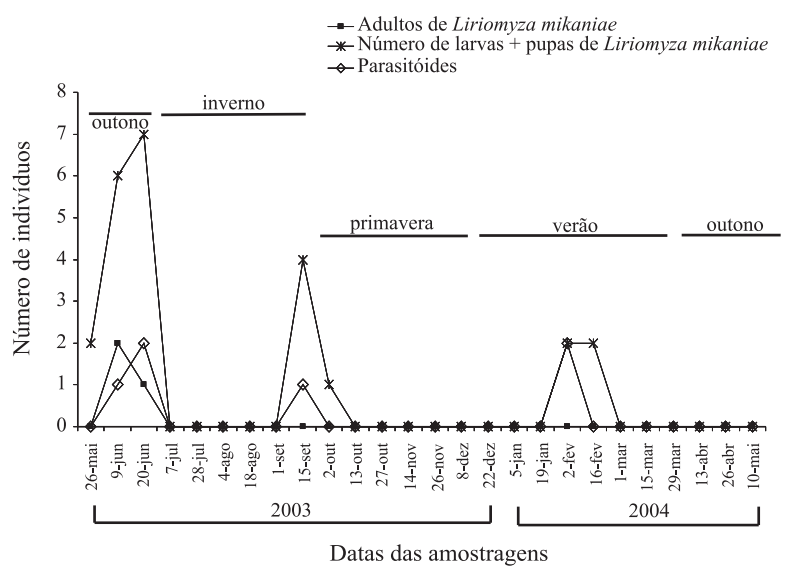

Fig. 5. Número de larvas + pupas de Liriomyza mikaniae amostradas em plantas de crescimento espontâneo, em pomar de tangor 'Murcott' e número de adultos de Liriomyza mikaniae e de parasitóides emergidos em laboratório, por ocasião de amostragem de maio de 2003 a maio de 2004, Montenegro, RS. 
espécies ainda não descritas. Os resultados também destacam o papel que as plantas de crescimento espontâneo podem ter como refúgio e/ou hábitat para seus inimigos naturais ou para hospedeiros destes. $\mathrm{O}$ manejo adequado da vegetação espontânea em pomares pode trazer como benefício a conservação de espécies benéficas, que poderão agir como agentes de controle natural daquelas consideradas pragas e auxiliar na preservação de uma maior biodiversidade em agroecossistemas.

Agradecimentos. Ao CNPq, pelas bolsas concedidas para o primeiro e segundo autores; ao Biólogo Luís Laux, por ter cedido o pomar para realização do estudo; ao Dr. Thomas Michael Lewinsohn (UNICAMP), pelo encaminhamento dos dípteros para identificação; a Dr ${ }^{\mathrm{a}}$. Graciela Valladares (Centro de Investigaciónes Entomológicas de Córdoba, Argentina), pela identificação dos dípteros minadores; ao Dr. Valmir Antônio Costa (Instituto Biológico de Campinas), pela confirmação das identificações de parasitóides; ao Dr. Christer Hansson (Department of Zoology, University of Lund, Suécia), pela identificação dos parasitóides de Chrysocharis, Neochrysocharis e Closterocerus; a Dra. Angélica Maria Penteado-Dias (UFSCar), pela identificação dos parasitóides de Opius; a Dra. Tânia Maria Guerra (UFSC), pela identificação dos parasitóides de Centistidea; ao Dr. Jorge Anderson Guimarães (EMBRAPA-CNPAT), pela identificação do parasitóide Agrostocynips clavatus; às bolsistas de Iniciação Científica (UFRGS), Rita de Cássia Antochevis e Ester Foelkel pela constante ajuda na realização dos trabalhos de campo e de laboratório.

\section{REFERÊNCIAS BIBLIOGRÁFICAS}

Ayres, M.; Ayres Júnior, M.; Ayres, D. L. \& Santos, A. S. Dos. 2000. BioEstat 2.0: aplicações estatísticas nas áreas das ciências biológicas e médicas. Belém, Sociedade Civil Mamirauá. 272p.

Barroso, G. M. \& Bueno, O. L. 2002. Compostas 5: subtribo Baccharidinae. In: ReItz, P. R. ed. Flora Ilustrada Catarinense. Itajaí, Herbário Barbosa Rodrigues. p.765-1065.

Begon, M. \& Mortimer, M. 1986. Population ecology: an unified study of animals and plants. Oxford, Blackwell Scientific. 219p.

Begon, M.; Harper, J. L. \& Townsend, C. R. 1990. Ecology: individuals, populations and communities. Oxford, Blackwell Science. 1068 p.

Byers, J. A. 2002. Leaf-mining insects. Disponível em: <http:/ /www. wcrl.ars.usda.gov/cec/insects/leafmine.htm > Acesso em: 27.12.2004.

Cabrera, A. L. \& Klein, R. M. 1989. Compostas. In: Reitz, P. R. ed. Flora Ilustrada Catarinense. Itajaí, Herbário Barbosa Rodrigues. p.649-750.

Carletti, E. 2004. Insectos de Argentina y del mundo. Disponível em: <http://www.axxon.com.ar/mus/info/artDondeHallarInsectos.htm> Acesso em: 27.12.2004.

Chávez, F. G. 2003. Moscas minadoras de Guatemala (Dip.; Agromyzidae; Liriomyza spp.). Disponível em:〈www.geocities.com/entomologia2003/pag_08.html> Acesso em: 02.02.2003.

Cure, J. R. \& Cantor, F. 2003. Atividade predadora e parasítica de Diglyphus begini (Ashm.) (Hymenoptera: Eulophidae) sobre Liriomyza huidobrensis (Blanch.) (Diptera: Agromyzidae) em cultivos de Gysophila paniculata L. Neotropical Entomology 32(1):85-89.

Gallo, D.; Nakano, O.; Silveira Neto, S.; Carvalho, R. P. L.; Baptista, G. C. De; Berti Filho, E.; Parra, J. R. P.; Zucchi, R. A.; Alves, S. B.; Vendramim, J. D.; Marchini, L. C.; Lopes, J. R. S. \& Омото, С. 2002. Entomologia Agrícola. Piracicaba, Fundação de Estudos Agrários Luiz de Queiroz. 920p.

Gauld, I. D. \& Hanson, P. E. The evolution, classification and identification of the Hymenoptera. In: HANson, P. E. \& Gauld, I. D. The Hymenoptera of Costa Rica. Reino Unido, Oxford University Press, 1995. p.138-156.

Hespenheide, H. A. 1991. Bionomics of leaf-mining insects. Annual Review Entomology 36:535-560.

Kissmann, K. G. \& Groth, D. 2000a. Plantas infestantes e nocivas. 2. ed. São Paulo, BASF Brasileira S.A., Indústrias Químicas. T.2, 978p.

. 2000b. Plantas infestantes e nocivas. 2. ed. São Paulo, BASF Brasileira S.A., Indústrias Químicas. T.3, 726p.

. 2000c. Plantas infestantes e nocivas. 2. ed. São Paulo, BASF Brasileira S.A., Indústrias Químicas. T.1. 825p.

León, A.; Pino, M. De Los A.; González, C. \& Pozo, E. Del. 2000. Evaluación comparativa de densidades de fitófagos y enemigos naturales en policultivo tomate-maíz. Cultivos Tropicales 21(1):53-60.

Patel, K. J.; Schuster, D. J. \& Smerage, G. H. 2003. Density dependent parasitism and host-killing of Liriomyza trifolli (Diptera: Agromyzidae) by Diglyphus intermediatus (Hymenoptera: Eulophidae). Florida Entomologist 86(1):8-14.

Pereira, D. I. da P.; Souza, J. C. DE; Santa-Cecília, L. V. C.; Reis, P. R. \& SouzA, M. DE A. 2002. Parasitismo de larvas da moscaminadora Liriomyza huidobrensis Blanchard (Diptera: Agromyzidae) pelo parasitóide Opius sp. (Hymenoptera: Braconidae) na cultura da batata com faixas de feijoeiro intercaladas. Ciência Agrotécnica 26(5):955-963.

Rodriguez, O.; Viégas, F.; Pompeu Júnior, J. \& Amaro, A. A. (coords). 1991. Citricultura brasileira. Campinas, Fundação Cargill. $492 \mathrm{p}$.

Schuster, D. J.; Gilreath, J. P.; Wharton, R. A. \& Seymour, P. R. 1991. Agromyzidae (Diptera) leafminer and their parasitoids in weeds associate with tomato in Florida. Environmental Entomology 20(2):720-723.

Smith, L. B. \& Downs, R. J. 1972. Amarantáceas. In: Reitz, P. R. ed. Flora Ilustrada Catarinense. Itajaí, Herbário Barbosa Rodrigues. p.1-110.

Southwood, T. R. E. 1978. Ecological methods: with particular reference to the study of insect populations. London, Chapman and Hall. 524p.

Spencer, K. A. 1996. Family Agromyzidae: Australasian/ oceanian diptera catolog web version. Diponível em: <www.hbs.bishopmuseum.org/aocat/agromyzidae.html> Acesso em: 02.02.2005.

Valladares, G. \& Salvo, A. 2001. Community dynamics of leafminers (Diptera: Agromyzidae) and their parasitoids (Hymenoptera) in a natural habitat from Central Argentina. Acta Oecologica 22:301-309.

Venette, R. C.; Hutchison, W. D.; Burkness, E. C. \& O’Rourke, P. K. 2003. El minador de la hoja de la alfafa: actualización de la investigación. Disponível em: <http:// wWw.ipmword.umn.edu/cancelado/spchapters/venettesp.htm> Acesso em: 10.10.2003.

W3Tropicos. Database of plant names, information on them, and sources of information. Disponível em: <http:// mobot.mobot.org/W3T/Search/vast.html > Acesso em: 27.12.2004. 